\title{
2MASS Star Counts and Galactic Structure
}

D. K. Ojha

Tata Institute of Fundamental Research, Mumbai, 400005 India

\begin{abstract}
This paper presents a global analysis of the first 2MASS (Two Micron All Sky Survey) data as observed in seven fields at different Galactic latitudes. These new data lead to strong constraints on the radial structure of the Galactic thin and thick disks. The interpretation of star counts and color distributions of stars in the near-infrared with a synthetic stellar population model provides strong evidence that the Galactic thin disk density scale length, $\mathrm{h}_{R}$, is rather short $(2.8 \pm 0.3 \mathrm{kpc})$. The Galactic thick disk population is revisited in the light of new data. We find the thick disk to have a local density of $3.5 \pm 2.0 \%$ of the thin disk, exponential scale height, $\mathrm{h}_{z}$, of $860 \pm 200 \mathrm{pc}$ and exponential scale length, $\mathrm{h}_{R}$, of $3.7_{-0.5}^{+0.8} \mathrm{kpc}$.
\end{abstract}

\section{Discussion}

Charley Lineweaver: What is the correlation between the scale height of the thick disk and constraints on mergers between our Galaxy and SMC- or LMC-type objects a few billion years ago or more?

Devendra Ojha: There are several recent determinations of galactic thick disk properties (Reid \& Majewski 1993, ApJ, 409, 635) that derive the gradient in the thick disk. If one supposes that the thick disk formation is due to mergers with other satellite galaxies, one may be able to produce the gradient in the scale height of the disk. 